\title{
Development of a calibration method for on-line analyzer of atmospheric particle mass concentrations
}

\author{
François Gaie-Levrel ${ }^{1, a}$, Charles Motzkus ${ }^{1}$, Tatiana Macé ${ }^{1}$, Christophe Debert $^{2}$, Christophe Ampe $^{2}$, Gérald Lacondemine $^{2}$ \\ ${ }^{1}$ Laboratoire National de métrologie et d'Essais (LNE), 1 rue Gaston Boissier, 75724 Paris Cedex 15, France \\ ${ }^{2}$ Airparif, Air quality monitoring network in Ile de France, 7 rue Crillion, 75004 Paris, France
}

\begin{abstract}
Résumé. Le TEOM-FDMS (Tapered Element Oscillating Microbalance with Filter Dynamics Measurement Systems) est un instrument de mesure très répandu au sein du réseau national de surveillance de la qualité de l'air. À l'heure actuelle, cet appareil est étalonné à l'aide de masses étalons raccordées au SI mais dont les valeurs et les différences de masse ne sont pas représentatives des masses particulaires atmosphériques prélevées. De plus, l'utilisation de ces masses étalons ne permet pas de prendre en compte un éventuel dysfonctionnement du système de prélèvement en amont de la mesure de la masse et du système de filtration intrinsèque au TEOM-FDMS. Par conséquent, une nouvelle méthode d'étalonnage a été développée pour les TEOM-FDMS de par la mise en place d'un système portable de génération de particules ayant des concentrations massiques connues et stables dans le temps. Cet article présente la caractérisation de la reproductibilité globale de ce générateur portable en terme de gamme de masses de référence, son couplage avec différents types de TEOM-FDMS et la comparaison globale entre les masses particulaires de référence définies et les masses lues obtenues pour chaque TEOM-FDMS impliqué dans cette étude.
\end{abstract}

\section{Introduction}

The French Central Laboratory for air quality monitoring (LCSQA), is identified as an expert and reference laboratory for the French Ministry of Ecology and for the French regional Air Quality Survey Networks (AQSNs) which measure the pollutants concentrations in ambient air, such as particulate matter (PM) measurements required by the 2008/50/CE European directive [1].

Actually, TEOM-FDMS (Tapered Element Oscillating Microbalance with Filter Dynamics Measurement Systems) [2, 3] instruments are mainly used in the field among the French PM analytical equipments. In order to ensure their efficient operation and the quality of the monitoring data with a proper QA/QC practice, a French protocol was developed for the flow rate control and calibration verification of TEOM-FDMS instruments $\left(\mathrm{K}_{0}\right.$ check, microbalance linearity) using three pre-weighed filters as calibration weight. Such calibration weights are characterized by masses around $100 \mathrm{mg}$, with a 5-10 mg mass differences between each of them. By taking into account a volumetric sampling flow rate of $3 \mathrm{~L} / \mathrm{min}$, the French $\mathrm{PM}_{10}$ limit value $\left(50 \mu \mathrm{g} / \mathrm{m}^{3}\right.$ daily average $)$ represents a particle mass of about $2 \mu \mathrm{g}$ in $15 \mathrm{~min}$ of sampling. The used calibration weight and their masses difference are then not completely representative of quarter hour atmospheric particle mass measurements. Moreover, these calibration weights do not take into account any technical problems of the sampling and microbalance filtration systems.
Therefore, in this paper we present the development and validation of a traceable and reproducible portable system for the FDMS-TEOM non-volatile particle mass calibration in a lower particle mass range compared to the calibrated weights. This system was developed to be easily used in AQSNs stations by taking into account the overall FDMS-TEOM sampling system in a complementary way compared to the actual protocol. This calibration method was developed with several constraints, such as (1) - generate and sample particles with known and stable concentrations over time, in the TEOM-FDMS filter by passing through its sampling system (without the sampling PM head) and in an external filter, (2) compare the TEOM-FDMS masses, read on the analyzer, with the reference masses determined by the gravimetric method using the external filter.

In this work, after presenting the developed portable system, we focus on the metrological validation of this particle mass calibration system in terms of repetability/reproducibility using the gravimetric reference method with downstream calibration tests performed on different FDMS-TEOM types.

\section{Material and method}

\subsection{Portable aerosol generation system}

For a mass calibration purpose, the best adapted generator was a constant output atomizer (TSI, model 3076) in terms of stable particle generation over time and

\footnotetext{
a Email de correspondance de l'auteur : francois.gaie-levrel@lne.fr
} 
of low filter clogging induced. Figure la presents the aerosol generation set-up which is composed of a portable air compressor connected to a filtered air supply system (TSI, model 3074B) upstream the atomizer inlet. Two diffusion driers (TSI, model 3062) were used downstream the atomizer outlet in order to remove water from particles, and a four-way valve allows its coupling with TEOM-FDMS. Figure $1 \mathrm{~b}$ shows a scheme of the coupling between the portable generator (in dotted line frame in fig. 1a and $1 \mathrm{~b}$ ) and a TEOM-FDMS using the four-way valve. Upstream a calibration, the valve is in position 1 (fig.1b) which implies the generated aerosol to go through an exhaust and allows the coupling between TEOM-FDMS and an HEPA filter. For the beginning of calibration, the valve is turning on position 2 (fig. $1 \mathrm{~b}$ ). In this configuration, the generated aerosol is sampled by the TEOM-FDMS. At the end of the calibration procedure, the valve is returned in its position 1 and the TEOM-FDMS filter is weighed using the double weighing method.

(a)

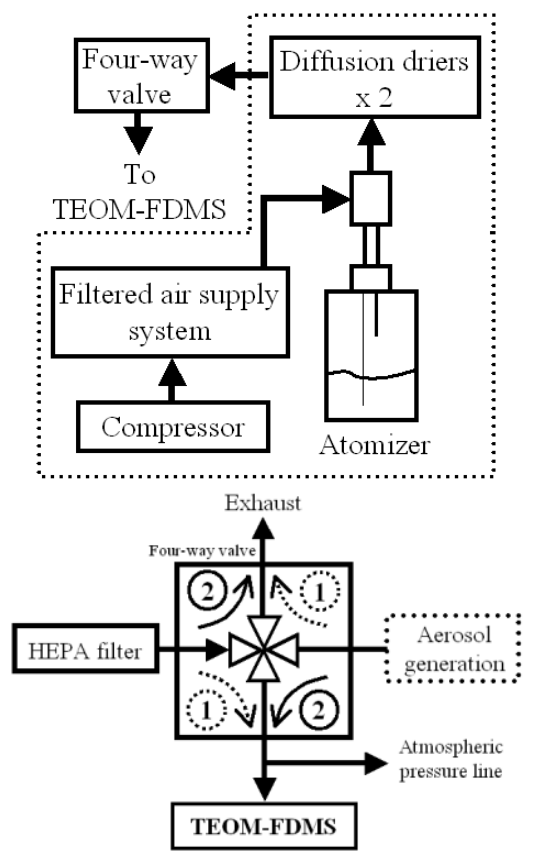

Figure 1. (a) Scheme of the portable aerosol generation system composed of a portable air compressor connected to a filtered air supply, an atomizer and two diffusion driers (dotted line frame) and of a four-way valve, (b) Description of the four-way valve used for the coupling between the portable aerosol generator and a TEOM-FDMS.

For this study, the $\mathrm{KCl}$ aerosol was generated by nebulizing a 0.1 g. $\mathrm{L}^{-1}$ aqueous solution of potassium chloride ( $\mathrm{KCl}$, AnalaR Normapur). A previous study of this project was dedicated to the set-up implementation by nebulizing a 4 g. $\mathrm{L}^{-1}$ potassium chloride solution [4]. This concentration was chosen to obtain a particulate loading of about few milligrams for a 30 min sampling time. The actual work, presented in this paper, concerns the procedure optimization of this calibration method by nebulizing a 0.1 g. $\mathrm{L}^{-1} \mathrm{KCl}$ solution in order to generate lower particulate masses, and then to be more representative of atmospheric measurements, i.e. below
$100 \mu \mathrm{g}$ for a $30 \mathrm{~min}$ sampling. To avoid any saturation process of the solution during its nebulization, a new 0.1 g. $\mathrm{L}^{-1}$ solution was made for each experiment presented in this paper. In order to characterize the produced aerosol in terms of number and mass size distributions, a Scanning Mobility Particle Sizer (SMPS composed by a differential mobility analyzer (DMA, model 3080, TSI) coupled to a condensation particle counter (CPC, model 3775, TSI)) was coupled to the portable aerosol generator in the same manner than a TEOM-FDMS. That characterization is presented in paragraph 3.1.

\subsection{Portable aerosol generator characterization with an external filter holder}

The characterization of the portable generator using an external filter holder consisted to sample the generated particles on an external filter and weighing it with a precision analytical balance in order to determine the particle mass generated on a given sample time. This gravimetric method allows (1) - to determine the generated particle masses on a given sampling time and to compare them to the TEOM-FDMS measurements, (2) - to determine the repeatability and reproducibility uncertainties in function of sampling time.

For doing such characterization, a $47 \mathrm{~mm}$ filter holder was coupled to the portable generator downstream the four-way valve instead of TEOMFDMS shown in Figures $1 \mathrm{a}$ and $1 \mathrm{~b}$. The same protocol described in paragraph 2.1 was used. Pallflex ${ }^{\circledR}$ filters, similar to those inserted in the TEOM-FDMS microbalance, i.e. Emfab ${ }^{\mathrm{TM}}$ TX40HI20-WW type constituted of borosilicate glass microfibers reinforced with a woven glass fabric and bonded with PTFE, were used. Downstream the filter holder, a mass flow controller (MFC) was used to regulate the sample flow at $(3.014 \pm 0.010) \mathrm{L} / \mathrm{min}$. This MFC was calibrated in comparison with a mass flow transfer standard Molbloc ${ }^{\mathrm{TM}}$ calibrated himself by a dynamic gravimetric method.

It is important to mention that this flow rate was voluntarily different from that associated with the standard method described in the NF EN 12341 standard [5], i.e. $2.3 \mathrm{~m}^{3} / \mathrm{h}(38 \mathrm{~L} / \mathrm{min})$, in order to be consistent with the TEOM-FDMS flow rate. Sampling time of $12 \mathrm{~min}, 24 \mathrm{~min}$ and $36 \mathrm{~min}$ were chosen consistently with the TEOM-FDMS operation by considering a $6 \mathrm{~min}$ rotation time of the FDMS valve. For each of the three sampling times, 108 experiments were performed on 12 days over four weeks using a new $\mathrm{KCl}$ solution for each experiment. This was achieved in order to obtain a global characterization of the portable generator by also taking into account its geographical displacement in the field. As for TEOM-FDMS filters, the external filters were weighed using the double weighing method.

\subsection{TEOM-FDMS implicated in this study}


Downstream the characterization of the portable generator with the external filter holder, the generation system was coupled with several TEOM-FDMS. This coupling consisted to generate $\mathrm{KCl}$ particles and to compare TEOM-FDMS masses, read on the analyzer, with masses determined using the external filter holder previously described, for identical generation parameters. It is important to notice that a previous study [4] showed that relative deviations between weighed and read TEOM-FDMS masses were comprised between 1 and $6 \%$. In this way, only read masses on TEOM-FDMS analyzers were taken into account for this work.

In this study, five TEOM-FDMS of the French AQSN for the capital city region, named Airparif, were involved, namely three at their laboratory:

- TEOM-FDMS n¹: TEOM 1400AB equipped with a $8500 \mathrm{C}$ FDMS module;

- TEOM-FDMS n²: TEOM 1400AB equipped with a $8500 \mathrm{~B}$ FDMS module with a type C dryer;

- TEOM-FDMS n³: TEOM-FDMS 1405F.

and two in urban stations:

- TEOM-FDMS n4: TEOM 1400AB equipped with a $8500 \mathrm{C}$ FDMS module;

- TEOM-FDMS n5: TEOM-FDMS 1405F.

These choice were defined in order to test different TEOM-FDMS types representative of the analytical park of the national survey network. In this way, two "old generation" TEOM types were used at the laboratory with two FDMS models. Such TEOM-FDMS was also used in the field and two "new generation" TEOM-FDMS were involved for laboratory and field studies. For coupling these instruments with the portable generator, the experimental set-up presented in Figures $1 \mathrm{a}$ and $1 \mathrm{~b}$ was used. Downstream the global characterization of the portable generator with the external filter holder, two experiments were performed for each of the five TEOMFDMS. For this study, the read masses on the TEOMFDMS analyzers were taken into account for $12 \mathrm{~min}$, $24 \mathrm{~min}$ and $36 \mathrm{~min}$ sampling times. For each of these experiments, new filters were used in TEOM microbalances and in FDMS modules, and a new $\mathrm{KCl}$ solution was also used. Associated results are presented in paragraph 3.3.

\section{Results and discussions}

\subsection{Characterization of the generated aerosol}

In order to characterize the aerosol generated from the nebulization of a 0.1 g.L $\mathrm{L}^{-1}$ solution, number and mass particle size distributions (PSD) measurements were performed using the SMPS previously described. Mass PSD were obtained from number PSD by taking into account a $1.98 \mathrm{~g} . \mathrm{cm}^{-3}$ density for $\mathrm{KCl}$. Number and mass PSD were respectively characterized by mean concentrations of $5.5 \quad$ x $10^{3} \quad \mu \mathrm{g} . \mathrm{cm}^{-3}$ and $1.1 \times 10^{7}$ particles. $\mathrm{cm}^{-3}$, median diameters of 47 and 128 $\mathrm{nm}$, mean diameters of 56 and $150 \mathrm{~nm}$, and modal diameters of 42 and $132 \mathrm{~nm}$.

\subsection{Determination of the global reproducibility of the portable generator with the external filter holder}

Table 1 presents the average weighed masses of the filter inserted in the external filter holder for sampling times of $12 \mathrm{~min}, 24 \mathrm{~min}$ and $36 \mathrm{~min}$ for 108 experiments performed on 12 days over four weeks. The geographical displacement of the portable generator in the field was also taken into account in that characterization. The associated repetability and reproducibility standard deviations ( $\mathrm{rSD}$ and RSD respectively) were calculated using the ISO 5725-2 standard [6].

The relative $\mathrm{rSD}$ and $\mathrm{RSD}$ are respectively comprised between $2.1 \%$ and $6.7 \%$ and between $3.0 \%$ and $9.5 \%$ for the first three weeks. The higher relative SD corresponds to the lower sampling time, i.e. $12 \mathrm{~min}$. This is also the case for the week $\mathrm{n}^{\circ} 4$ in which the relative $\mathrm{rSD}$ and RSD are respectively comprised between $5.4 \%$ and $16.2 \%$ and between $6.4 \%$ and $16.2 \%$.

\begin{tabular}{|c|c|c|c|c|}
\hline & \\
\hline & & $12 \mathrm{~min}$ & $24 \mathrm{~min}$ & $36 \mathrm{~min}$ \\
\hline \multicolumn{2}{|c|}{ Week $n^{\circ} 1-$ Average value ( $\left.\mu g\right)$} & 39.1 & 67.1 & 97.9 \\
\hline \multirow{2}{*}{ rSD } & Absolute $(\mu \mathrm{g})$ & 2.6 & 2.9 & 2.7 \\
\hline & Relative & $6.7 \%$ & $4.3 \%$ & $2.8 \%$ \\
\hline \multirow{2}{*}{ RSD } & Absolute ( $\mu \mathrm{g})$ & 2.9 & 4.2 & 5.3 \\
\hline & Relative & $7.4 \%$ & $6.2 \%$ & $5.4 \%$ \\
\hline \multicolumn{2}{|c|}{ Week $n^{\circ} 2-$ Average value $(\mu g)$} & 32.7 & 66.0 & 96.9 \\
\hline \multirow{2}{*}{ rSD } & Absolute $(\mu \mathrm{g})$ & 1.5 & 2.9 & 2.0 \\
\hline & Relatif & $4.7 \%$ & $4.5 \%$ & $2.1 \%$ \\
\hline \multirow{2}{*}{ RSD } & Absolute $(\mu \mathrm{g})$ & 1.9 & 2.9 & 2.9 \\
\hline & Relative & $5.8 \%$ & $4.5 \%$ & $3.0 \%$ \\
\hline \multicolumn{2}{|c|}{ Week $n^{\circ} 3$ - Average value (ug) } & 32.4 & 61.4 & 96.3 \\
\hline \multirow{2}{*}{ rSD } & Absolute $(\mu \mathrm{g})$ & 2.2 & 2.6 & 3.3 \\
\hline & Relative & $6.6 \%$ & $4.3 \%$ & $3.4 \%$ \\
\hline \multirow{2}{*}{ RSD } & Absolute $(\mu \mathrm{g})$ & 3.1 & 2.6 & 3.5 \\
\hline & Relative & $9.5 \%$ & $4.3 \%$ & $3.6 \%$ \\
\hline \multicolumn{2}{|c|}{ Week $n^{\circ} 4$ - Average value ( $\mu g$ ) } & 29.6 & 61.5 & 89.1 \\
\hline \multirow{2}{*}{ rSD } & Absolute $(\mu \mathrm{g})$ & 4.8 & 5.4 & 4.8 \\
\hline & Relative & $16.2 \%$ & $8.8 \%$ & $5.4 \%$ \\
\hline \multirow{2}{*}{ RSD } & Absolute $(\mu \mathrm{g})$ & 4.8 & 5.7 & 5.7 \\
\hline & Relative & $16.2 \%$ & $9.3 \%$ & $6.4 \%$ \\
\hline \multicolumn{2}{|c|}{ Global average value $(\mu \mathrm{g})$} & 33.5 & 64.0 & 95.0 \\
\hline \multirow{2}{*}{$\begin{array}{c}\text { Global } \\
\text { rSD }\end{array}$} & Absolute $(\mu \mathrm{g})$ & 4.2 & 3.6 & 5.2 \\
\hline & Relative (\%) & $12.6 \%$ & $5.6 \%$ & $5.5 \%$ \\
\hline \multirow{2}{*}{$\begin{array}{c}\text { Global } \\
\text { RSD }\end{array}$} & Absolute ( $\mu \mathrm{g})$ & 4.2 & 3.6 & 5.2 \\
\hline & Relative (\%) & $12.6 \%$ & $5.6 \%$ & $5.5 \%$ \\
\hline
\end{tabular}

Table 1. Average weighed masses of the filters inserted in the external filter holder for 12, 24 and 36min sampling times performed on four weeks.

In a global point of view, this characterization allows determining the global reproducibility of the portable generator. The reference masses for each of the three sampling times are equal to $(34 \pm 9) \mu \mathrm{g}$; 
$(64 \pm 8) \mu g$; and $(95 \pm 11) \mu g$ for sampling times of $12 \mathrm{~min}, 24 \mathrm{~min}$ and $36 \mathrm{~min}$ respectively. These values correspond to the average of the four masses for a given sampling time, while the associated uncertainties correspond to two times the $\mathrm{RSD}(\mathrm{k}=2)$ recalculated by following the ISO 5725-2 standard. As mentioned before, this work represents a step forward concerning the representativity of the generated particulate masses for atmospheric measurements.

\subsection{Comparisons between TEOM-FDMS read masses and external filter holder masses}

As mentioned before, the reference masses obtained by using the external filter holder allow comparing TEOM-FDMS masses with gravimetric characterization of the portable generator. Figure 2 presents a comparison between the TEOM-FDMS read masses with reference masses defined as an identical grey reference zone for each graphic of Figure 2. This grey reference zone was defined by the global reproducibility of the portable generator presented in paragraph 3.2 (see Table 1 for data). For each TEOM-FDMS implicated in this study, i.e. for each graph of Figure 2, two experiments were performed. The TEOM-FDMS masses were read on each analyzer for sampling times of $12 \mathrm{~min}, 24 \mathrm{~min}$ and 36 min and were multiplied by a factor 2 due to the intrinsic working of a TEOM-FDMS ( 6 min on the base and $6 \mathrm{~min}$ on the reference). The associated type $B$ uncertainties were calculated by following the FD X43-070-6 guide [7].

Concerning laboratory measurements (TEOMFDMS $n^{\circ} 1,2$ and 3 ) and field measurements (TEOM-FDMS $\mathrm{n}^{\circ} 4$ and 5 ), relative deviations between reference masses determined with the external filter holder and TEOM-FDMS read masses are comprised between $0.5 \%$ and $13.8 \%$ and between $1.1 \%$ and $12.9 \%$ for laboratory and field measurements respectively. By taking into account the expanded uncertainties for reference masses (grey reference zone) and the uncertainties associated to the TEOM-FDMS read masses, an overlapping of the TEOM-FDMS measurements with the reference zone for TEOM-FDMS $n^{\circ} 1,2$ and 5 was obtained for each of the three sampling times. Each of these measurements were therefore validated.

In the case of a non-overlapping of measurements with the reference zone, further technical investigations should have been conducted in order to explain such phenomenon, probably caused by a technical failure for example. Concerning TEOMFDMS $n^{\circ} 3$ a non-linear response was obtain for one of both experiments at the sampling time of $36 \mathrm{~min}$ (purple triangles). This measurement can not be validated due to the fact that no overlapping is obtain even if the associated uncertainty is taken into account. This can be explained by the fact that new generation TEOM-FDMS 1405F presents a more important instability in comparison with older versions in terms of filter loading signals [8]. For TEOM-FDMS $n^{\circ} 4$, the measurement at $36 \mathrm{~min}$ for experiment $n^{\circ} 1$ is also out of the reference zone. However, by taking into account the associated type $B$ uncertainty, this measurement can be considered as valid. 

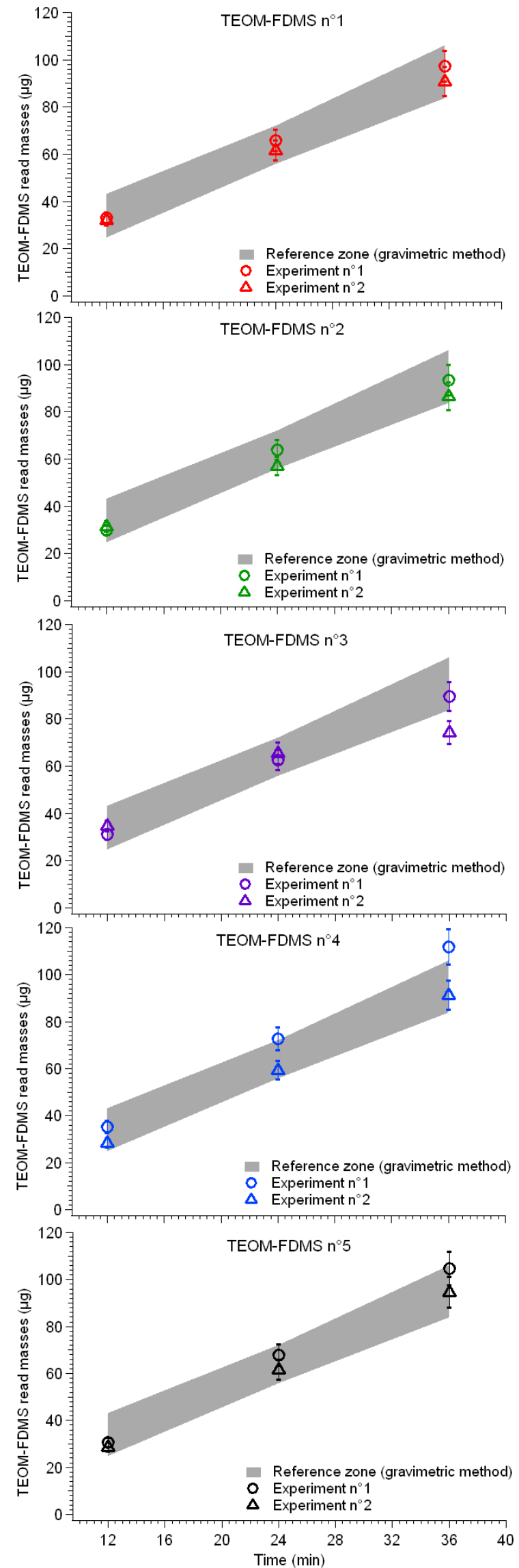

Figure 2. Comparisons between read masses for each of the five TEOM-FDMS with reference masses zone determined with the external filter holder.

\section{Conclusion and outlooks}

This study was dedicated to the development of a calibration method for on-line analyzer of particle mass concentrations in ambient air, such as TEOMFDMS, consisting in (1) - generating and sampling particles with known and stable concentrations over time, in the TEOM-FDMS filter by passing through its sampling system (without the sampling head) and in an external filter, (2) comparing the TEOM-FDMS masses read on the analyzer with the reference masses determined by the gravimetric method using the external filter.

Moreover, this method was developed by taking into account the overall FDMS-TEOM sampling system in a complementary way compared to the actual protocol and for lower particulate masses than calibration weight actually used.

For this work, a reference masses zone was determined thanks to measurements with an external filter holder and calculated from 108 experiments performed on 12 days. The reference masses obtained at sampling times of $12 \mathrm{~min}, 24 \mathrm{~min}$ and $36 \mathrm{~min}$ with the expanded reproducibility standard deviations $(\mathrm{k}=2)$ calculated in accordance with the ISO 5725-2 standard, are respectively equal to $(34 \pm 9) \mu \mathrm{g} ;(64 \pm 8) \mu \mathrm{g}$; and $(95 \pm 11) \mu \mathrm{g}$.

Downstream that characterization, the portable generator was coupled to three TEOM-FDMS of the French AQSN for the capital city region, named Airparif, at their laboratory and to two other in urban stations. A global comparison between the previously defined reference masses zone and TEOM-FDMS read masses was performed for each of these instruments. Relative deviations between reference masses determined with the external filter holder and TEOM-FDMS read masses were comprised between $0.5 \%$ and $13.8 \%$ and between $1.1 \%$ and $12.9 \%$ for laboratory and field measurements respectively. From this comparison, the majority of the measurements were validated with the gravimetric measurements, except for one point for a new TEOM-FDMS generation.

By considering the AQSN specificities to have a portable generator with an easier field use for TEOM-FDMS calibration, a miniaturization of the actual portable generator is currently in development at LNE. This will lead to an easily portable system due to its lightweight and to a selfgoverning system without the need of an electrical source. This miniaturized generator will also be developed in order to apply the calibration method to beta gauges, increasingly used in the French air quality survey network, leading to a versatile portable system.

\section{References}

1. Directive 2008/50/EC of the European Parliament and of the Council of 21 May 2008 on ambient air 
quality and cleaner air for Europe, Official J., L152, 1-44 (2008)

2. Patashnick, H. and Rupprecht, E.G., J. Air Waste Manage. Assoc., 41, 1079-1083 (1991)

3. Meyer, M.B., Patashnick, H., Ambs, J.L., "Ongoing development of a continuous reference standard particulate matter mass monitor for ambient air", presented at the Symposium on Air Quality Measurement Methods and Technology, San Fransisco, CA, Pittsburg, USA, Air and Waste Manage. Assoc. (2002)

4. Gaie-Levrel, F., Motzkus, C., Macé, T., VaslinReimann, S., "Développement d'une méthode d'étalonnage pour les instruments de mesure de concentration massique particulaire en air ambiant", Revue Française de Métrologie, 38-2 (2015)

5. NF EN 12341 standard, " Air ambiant - Méthode normalisée de mesurage gravimétrique pour la détermination de la concentration massique MP10 ou MP2,5 de matière particulaire en suspension" (2014)

6. NF ISO 5725-2, "Accuracy (trueness and precision) of measurement methods and results -- Part 2: Basic method for the determination of repeatability and reproducibility of a standard measurement method" (1994)

7. FD X43-070-6, «Qualité de l'air - Guide pratique pour l'estimation de l'incertitude de mesure des concentrations en polluants dans l'air ambiant »Partie 6 «Estimation des incertitudes sur les concentrations massiques de particules mesurées en automatique » (2011)

8. Verlhac S., « Guide méthodologique pour la surveillance des $\mathrm{PM}_{10}$ et $\mathrm{PM}_{2,5}$ par TEOM-FDMS dans l'air ambiant », Rapport LCSQA (2013) 